Kardiologe 2017 · 11:355

DOI 10.1007/s12181-017-0163-z

Online publiziert: 19. Juni 2017

๑) Springer Medizin Verlag GmbH 2017

CrossMark

\section{Originalpublikation}

Calkins H, Willems S, Gerstenfeld EP et al (2017) Uninterrupted dabigatran versus warfarin for ablation in atrial fibrillation. $N$ Engl J Med 376:1627-1636

Elektrophysiologische Interventionen inklusive Aggregatimplantationen und Katheterablationen werden bei Patienten mit VHF standardmäßig unter fortgesetzter therapeutischer Antikoagulation mit Vitamin-K-Antagonisten durchgeführt. Hierdurch lassen sich sowohl Blutungs- als auch thrombembolische Komplikationen reduzieren. Unklarheit herrscht, wie mit direkten oralen Antikoagulanzien (DOAK) umgegangen werden soll, da es bisher nur eine kontrollierte Untersuchung (VENTUREAF) gab.

\section{Zusammenfassung der Studie}

In einer globalen multizentrischen, prospektiv randomisierten Studie an 104 Zentren wurde bei 635 Patienten eine VHFAblation durchgeführt. In der Kontrollgruppe wurden die Patienten 4 bis 8 Wochen vor und 8 Wochen nach der Ablation mit Warfarin behandelt (Ziel-INR 2-3). In der Verumgruppe wurde 2-mal täglich $150 \mathrm{mg}$ Dabigatran für den gleichen Zeitraum verabreicht. Am Tag der Intervention wurde die Studienmedikation nicht unterbrochen. Primärer Endpunkt der Studie war das Auftreten von MajorBlutungen (ISTH). Das Patientenkollektiv war jung (mittleres Alter $59 \pm 10$ Jahre) und hatte in ca. zwei Drittel der Fälle paroxysmales VHF. Das thrombembolische Risiko (Chads-Vasc-Score = 2) war niedrig. Im Studienzeitraum traten signifikant weniger Major-Blutungen in der

\title{
Boris Schmidt
}

Cardioangiologisches Centrum Bethanien (CCB), Frankfurt am Main, Deutschland

\section{VHF-Ablation ohne Unterbrechung von Dabigatran}

Dabigatran-Gruppe auf (5 [1,6\%] vs. 22 [6,9\%]; relative Risikoreduktion 77,2\%). Es wurde nur ein thrombembolisches Ereignis unter Warfarin beobachtet.

\section{Kommentar}

Der überwiegende Teil der Patienten, die sich heutzutage zu einer Katheterablation von VHF vorstellen, wird mit DOAKs behandelt. Die Ergebnisse der vorliegenden Studie liefern daher ein wichtiges Puzzleteil für den Umgang mit DOAKs im klinischen Alltag. Die ununterbrochene Gabe des direkten Thrombininhibitors Dabigatran scheint im Rahmen von VHF-Ablationen sicher im Hinblick auf das Auftreten schwerer Blutungen zu sein. Allerdings muss einschränkend angemerkt werden, dass keine der vorliegenden Blutungen so schwerwiegend war, dass der monoklonale Antikörper Idarucizumab zum Einsatz kommen musste. Diesbezüglich sollten folglich die Daten der Registerbeobachtungen abgewartet werden.

Die Autoren geben zu bedenken, dass die Studie ohne Nullhypothese geplant wurde, d.h. lediglich beschreibender Natur ist, da aufgrund der niedrigen Inzidenzen des primären Endpunktes befürchtet werden musste, eine zu große Anzahl an Patienten einschließen zu müssen. Dennoch ist aus den Ergebnissen ableitbar, dass prozedurale Blutungskomplikationen (Leiste, Perikarderguss etc.) unter Dabigatran gut beherrschbar sind.

Die Frage nach der äquipotenten Wirksamkeit, nämlich die Vermeidung thrombembolischer Komplikationen im Rahmen der Ablation kann nicht vollständig beantwortet werden. Erfreulicherweise trat kein derartiges Ereignis im Dabigatran-Arm auf, doch die Zahl der analysierten Patienten ist naturgemäß zu gering. Für den klinischen Alltag bleibt jedoch die Erkenntnis, dass ein mit Dabigatran antikoagulierter Patient die Therapie nicht unterbrechen muss oder gar komplizierte Bridging-Maßnahmen mit subkutan applizierten, niedermolekularen Heparinen durchführen muss. Trotz kleiner methodischer Mängel wird die RE-CIRCUIT-Studie das Management von Patienten, die sich einer VHFAblation unterziehen, weiter vereinfachen.

\section{Korrespondenzadresse}

\section{PD Dr. B. Schmidt}

Cardioangiologisches Centrum Bethanien (CCB)

Im Prüfling 23, 60389 Frankfurt am Main, Deutschland

b.schmidt@ccb.de

Interessenkonflikt. B. Schmidt gibt an, dass kein Interessenkonflikt besteht. 